\title{
Risco de câncer no Brasil: tendências e estudos epidemiológicos mais recentes
}

\author{
The risk of cancer in Brazil: tendencies and recent epidemiologic studies
}

\author{
Maximiliano Ribeiro Guerra, ${ }^{1}$ Cláudia Vitória de Moura Gallo, ${ }^{2}$ Gulnar Azevedo e Silva Mendonça ${ }^{3}$
}

\section{Resumo}

O número de casos de câncer tem aumentado de maneira considerável em todo o mundo, principalmente a partir do século passado, configurando-se, na atualidade, como um dos mais importantes problemas de saúde pública mundial. A freqüência de distribuição dos diferentes tipos de câncer apresenta-se variável em função das características de cada região, o que enfatiza a necessidade do estudo das variações geográficas nos padrões desta doença, para seu adequado monitoramento e controle. Por meio de revisão da literatura especializada mais recente, foram descritos alguns dos principais tipos de câncer no Brasil, com enfoque nas pesquisas epidemiológicas que investigaram os fatores associados ao risco de adoecer por estes cânceres em nosso país. Desta forma, os autores deste estudo objetivaram contribuir para a avaliação do risco atual de câncer no Brasil, buscando uma análise das tendências em sua incidência, estratégia de fundamental valor para o desenvolvimento de políticas de saúde adequadas que visem ao controle desta doença em nosso país.

Palavras-chave: Neoplasias; Epidemiologia; Fatores de risco; Incidência; Brasil.

\section{Abstract}

The number of cancer cases has been increasing all over the world, mainly since the last century, to the point where nowadays it is one of the most serious public health problems worldwide. The frequency of distribution of the various types of cancer varies according to the specific characteristics of each region, which implies a need to study how geographic variations impact on this disease aiming an adequate monitoring and control. This work is a review of the most recent specialized literature, describingsome of the main types of cancer in Brazil have been described, focusing on epidemiologic research which has studied the risk factors associated with these cancers in our country. In this way, the authors aimed to contribute to an evaluation of the present risk of cancer in Brazil, analyzing the tendencies of its incidence, that could help to develop adequate public health policies to control this disease in our country.

Key words: Neoplasms; Epidemiology; Risk factors; Incidence; Brazil.

\footnotetext{
${ }^{1}$ Mestre e Doutorando em Epidemiologia pelo Instituto de Medicina Social, UERJ. Professor do Departamento de Saúde Coletiva da Faculdade de Medicina, Universidade Federal de Juiz de Fora.

${ }^{2}$ Doutora em Biologia Molecular pela Universidade Paris VII. Professora do Departamento de Biologia Celular e Genética, Instituto de Biologia Roberto Alcântara Gomes, UERJ.

${ }^{3}$ Doutora em Medicina Preventiva pela Faculdade de Medicina da Universidade de São Paulo. Coordenadora da CONPREV, Instituto Nacional de Câncer. Professora do Departamento de Epidemiologia do Instituto de Medicina Social, UERJ.

Enviar correspondência para: Maximiliano Ribeiro Guerra. Rua Espírito Santo 969/1001, Centro; 36010-041 Juiz de Fora, MG. E-mail: guerramr@hotmail.com
} 


\section{INTRODUÇÃO}

O câncer é um importante problema de saúde pública em países desenvolvidos e em desenvolvimento, sendo responsável por mais de seis milhões de óbitos a cada ano, representando cerca de $12 \%$ de todas as causas de morte no mundo. Embora as maiores taxas de incidência de câncer sejam encontradas em países desenvolvidos, dos dez milhōes de casos novos anuais de câncer, cinco milhôes e meio são diagnosticados nos países em desenvolvimento. ${ }^{1}$

O processo global de industrialização, ocorrido principalmente no século passado, conduziu a uma crescente integração das economias e das sociedades dos vários países, desencadeando a redefinição de padrões de vida com uniformização das condiçôes de trabalho, nutrição e consumo. ${ }^{2}$ Paralelamente, deu-se uma significativa alteração na demografia mundial, devido à redução nas taxas de mortalidade e natalidade com aumento da expectativa de vida e envelhecimento populacional.

Este processo de reorganização global determinou grande modificação nos padróes de saúde-doença no mundo. Tal modificação, conhecida como transição epidemiológica, foi caracterizada pela mudança no perfil de mortalidade com diminuição da taxa de doenças infecciosas e aumento concomitante da taxa de doenças crônico-degenerativas, especialmente as doenças cardiovasculares e o câncer. ${ }^{3,4}$ Esta transformação do perfil epidemiológico das populações vem tornando-se, ao longo dos anos, cada vez mais complexa e de difícil entendimento, em função do aparecimento de novas doenças e o ressurgimento de antigos agravos à saúde Aids/HIV, malária, dengue, tuberculose, entre outros no cenário da saúde pública mundial., ${ }^{2,5}$

Nos países da América Latina, ao contrário dos países desenvolvidos, esta transição epidemiológica ainda não se completou, observando-se um aumento na ocorrência de doenças crônico-degenerativas, enquanto a freqüência de doenças infecciosas e de doenças transmissíveis por vetor biológico - como malária e dengue - permanecem elevadas, além da presença constante de desnutrição. ${ }^{3,4}$ Atualmente, considera-se a América Latina como a mais urbanizada das regiōes menos desenvolvidas do mundo, sendo que esta urbanização tem sido acompanhada de pobreza urbana maciça, o que tem contribuído para o agravamento das disparidades sociais. ${ }^{6}$ Deve-se levar em consideração, também, a repercussão da rápida mudança na condição nutricional desta região, desencadeada pelo processo de industrialização, o que afetou, sobremaneira, a prevalência de doenças crônicas como o câncer, doenças cardiovasculares, diabetes tipo 2, doença de Alzheimer e outros agravos relacionados ao envelhecimento e à obesidade. ${ }^{2,7}$

Em virtude das desigualdades sociais existentes na América Latina, o mapa global de distribuição dos tipos de câncer nesta região segue uma superposição semelhante à encontrada no perfil de morbimortalidade anteriormente mencionado. Neste contexto, o Brasil destaca-se como uma área interessante para monitoramento e controle das tendências na incidência de câncer, assim como para estudo das variações geográficas nos padrôes desta doença.

Neste trabalho, é apresentada uma revisão da literatura recente sobre risco de câncer no Brasil, descrevendo alguns dos principais tipos de câncer no país, com enfoque nas pesquisas epidemiológicas que investigaram os fatores associados ao risco de adoecer por estes cânceres. Desta maneira, os autores buscaram contribuir para uma melhor compreensão dos fatores que determinam maior incidência de câncer em nosso país.

\section{MATERIAL E MÉTODOS}

A revisão de artigos foi realizada através da consulta ao Index Medicus Medline (http://www.ncbi.nlm.nih.gov/ pubmed), considerado como critério inicial para seleção. Optou-se por esta base de dados por ser a mesma uma das principais fontes de publicações científicas na atualidade e, a partir de seu sistema de busca, utilizouse as palavras-chave "cancer incidence" e "Brazil" e também "cancer risk" e "Brazil". Foram selecionados apenas os artigos nas línguas inglesa, portuguesa e espanhola publicados no período compreendido entre 01/01/00 e 30/06/04. Realizou-se leitura cuidadosa de todos os artigos selecionados, incluindo, neste estudo, aqueles que utilizaram métodos epidemiológicos na abordagem do risco de câncer no país. Foram também incluídas publicações de órgãos oficiais sobre distribuição e freqüência do câncer no âmbito nacional e internacional, assim como outros artigos originais restritos ou não ao intervalo pré-estabelecido de seleção dos demais, em decorrência de seu valor como referência histórica e/ou sua relevância no contexto apresentado.

\section{CARGA DE CÂNCER NO BRASIL}

A distribuição epidemiológica do câncer no Brasil sugere uma transição em andamento, envolvendo um aumento entre os tipos de câncer normalmente associados a alto status sócio-econômico - câncer de mama, próstata e cólon e reto - e, simultaneamente, a 
presença de taxas de incidência persistentemente elevadas de tumores geralmente associados com a pobreza - câncer de colo de útero, pênis, estômago e cavidade oral.8 Esta distribuição certamente resulta de exposição a um grande número de diferentes fatores de risco ambientais relacionados ao processo de industrialização - agentes químicos, físicos e biológicos - e de exposição a outros fatores relacionados às disparidades sociais.

De acordo com os dados de dez registros de câncer de base populacional do Brasil," os tumores mais freqüentes no país são próstata, pulmão, estômago, cólon e reto e esôfago na população masculina. Em mulheres, predomina o câncer de mama, seguido pelos cânceres de colo uterino, cólon e reto, pulmão e estômago. Vale destacar que, segundo a Organização Mundial de Saúde (2002), ${ }^{1}$ sobressaem-se, entre os cinco tipos de câncer mais freqüentes, os tumores de pulmão, de cólon e reto e de estômago, tanto nos países industrializados, quanto nos países em desenvolvimento. Com relação ao sexo, a prevalência de câncer entre homens e mulheres é muito similar nos países desenvolvidos, enquanto nos países em desenvolvimento, a prevalência nas mulheres é $25 \%$ maior, o que reflete o predomínio, em homens, de localizações de câncer com pior sobrevida, tais como fígado, esôfago e estômago. ${ }^{10}$

As principais causas de morte por câncer no Brasil em 2001 foram os tumores de pulmão, próstata, estômago, esôfago e boca e faringe em homens e os tumores de mama, pulmão, cólon e reto, colo de útero e estômago em mulheres. ${ }^{11}$ Vale comentar que, em estudo conduzido para analisar a mortalidade por câncer no Brasil no período compreendido entre 1980 e 1995, foram constatados como principais causas de óbito o câncer de pulmão em homens e o câncer de mama em mulheres, com risco de morte aumentado nas regiōes Sul e Sudeste, as mais desenvolvidas do país. ${ }^{12}$

\section{Câncer de pulmão}

O câncer de pulmão é o câncer mais comum no mundo e, também, a principal causa de morte por neoplasia maligna. ${ }^{13}$ Sua ocorrência está associada ao uso de tabaco e a demonstração desta relação configurase como uma das mais importantes vitórias da Epidemiologia. ${ }^{6} \mathrm{O}$ tabagismo trata-se de um importante problema de saúde pública na América Latina, que apresenta também grande número de casos de câncer de pulmão, apesar desta patologia ser característica de países desenvolvidos. ${ }^{14}$ No Brasil, as taxas de incidência bruta e ajustada para câncer de pulmão estão aumentando, especialmente entre as mulheres, devido, principalmente, a aceleração no consumo do tabaco e a difusão do tabagismo na população feminina, sendo a incidência e a mortalidade em homens no estado do Rio Grande do Sul comparáveis aos dados dos Estados Unidos. ${ }^{15}$ Em pesquisa baseada em três estudos casocontrole realizados em cidade de médio porte do nosso país, ${ }^{16}$ foram evidenciados, em pacientes com câncer de pulmão, risco populacional atribuível ao fumo de $63 \%$ para ex-fumantes e de $71 \%$ para fumantes. Em Porto Alegre, encontram-se as maiores taxas de incidência ajustadas por idade do país, 54,9/100.000 em homens e 16,1/100.000 em mulheres.'

$\mathrm{O}$ tabagismo contribui não somente para o aumento da carga de câncer de pulmão em nosso país, mas também para o aumento da incidência de outros tipos de câncer, tais como de laringe, esôfago, boca e faringe, os dois últimos principalmente se associado a consumo de álcool e precárias condições de nutrição, outros fatores de risco muito comuns no Brasil. ${ }^{16,17}$

\section{Câncer de boca e de faringe}

A incidência de câncer de boca e de faringe tem aumentado no mundo nas últimas décadas, acompanhando o aumento no consumo de tabaco e de bebidas alcoólicas. Em Porto Alegre, foram observadas altas taxas de incidência de câncer de boca, ajustadas por idade pela população mundial, em ambos os sexos (8,3/100.000 em homens e 1,4/100.000 em mulheres), encontrando-se entre as mais elevadas do mundo. ${ }^{18} \mathrm{Em}$ estudo conduzido para investigar a ocorrência de câncer de boca e de faringe em população residente nas cinco regiōes do Brasil, verificou-se que a mais alta taxa de incidência, ajustada por idade pela população mundial, destes dois tipos de câncer combinados, encontrava-se em São Paulo, na região Sudeste do país (25,3/100.000 em homens e 4,9/100.000 em mulheres), achado semelhante a outras regiōes do mundo que apresentavam alta incidência destes cânceres. ${ }^{17}$ Cabe comentar que a mortalidade do câncer de boca em São Paulo permaneceu estacionada em níveis elevados durante o período de 1980 a $1998 .{ }^{19}$ Dados mais recentes do Ministério da Saúde (2003) ${ }^{9}$ demonstram que os maiores valores das taxas anuais de incidência, ajustadas por idade, de câncer de boca encontram-se, na população masculina, em São Paulo $(7,6 / 100.000)$ e, na população feminina, em Natal (3,3/100.000).

\section{Câncer de esôfago}

Os fatores de risco para o câncer de esôfago são similares aos descritos para o câncer de boca e de laringe. Estudos apontam o consumo de tabaco, de álcool e de 
mate como importantes e independentes fatores de risco para o câncer do trato aerodigestivo superior nos países da América Latina, ${ }^{20-23}$ levando-se em consideração a presença de interação e fatores de confusão, tais como a temperatura da bebida - em algumas áreas, o mate é consumido muito quente - e os hábitos alimentares como o churrasco, muito comuns na regiāo Sul do Brasil. Porto Alegre (15,4/100.000 em homens e 4,5/100.000 em mulheres) e Distrito Federal (14,7/100.000 em homens e 4,5/100.000 em mulheres) apresentam as mais altas taxas anuais de incidência, ajustadas por idade, de câncer de esôfago no país. ${ }^{9}$

\section{Câncer de estômago}

O risco de câncer de estômago relaciona-se a hábitos dietéticos tais como consumo de aditivos alimentares e de elevado teor de sal, que ocasionam inflamação da mucosa gástrica, além de associar-se à infecção por $H$. pylori. ${ }^{6} \mathrm{O}$ Japão apresenta a mais alta taxa de incidência deste tipo de câncer no mundo (59,9 a 91,6/100.000 em homens e 23,8 a 38,9/100.000 em mulheres). ${ }^{24} \mathrm{~A}$ incidência de câncer de estômago em imigrantes japoneses residentes em São Paulo excede àquela encontrada nos brasileiros não japoneses em cerca de $30 \%$, sendo pouco menor que a incidência observada nos japoneses residentes no Japão. Tal achado não foi observado em imigrantes japoneses residentes nos Estados Unidos da América, que apresentaram uma considerável redução na incidência desta patologia após a migração. ${ }^{25} \mathrm{~A}$ introdução do hábito de consumo diário de carne por parte dos imigrantes japoneses e de seus descendentes em nosso país pode estar associada ao risco de câncer de estômago nesta população, na qual destaca-se também o efeito protetor do consumo de frutas. ${ }^{26}$ No Brasil, as maiores taxas anuais de incidência, ajustadas por idade, foram encontradas em São Paulo (38,8/100.000 em homens e 15,0/100.000 em mulheres) e Distrito Federal (32,7/100.000 em homens e 14,7/ 100.000 em mulheres). ${ }^{9}$ Baixo nível sócio-econômico, tabagismo e baixo consumo de frutas e de vegetais foram considerados como fatores de risco para o câncer de estômago em São Paulo. ${ }^{27}$ Verdecchia et al. $(2002)^{28}$ avaliaram a sobrevida do câncer gástrico em registros de base populacional em cidades de quatro continentes, incluindo o registro de Campinas, e concluíram que as grandes diferenças observadas entre tais áreas foram quase que exclusivamente devidas aos diferentes tipos de casos de câncer de estômago, destacando-se a importância do estádio da doença como um indicador do efeito do diagnóstico tardio sobre o prognóstico dos pacientes acometidos.

\section{Câncer de colo de útero}

A história natural do câncer de colo de útero também está fortemente relacionada à presença de infecção, sendo a associação deste com o papilomavírus humano (HPV) muito bem documentada na atualidade. ${ }^{29-31}$ Além disso, outros fatores de risco para esta doença já foram descritos, como por exemplo o número de parceiros sexuais e o tabagismo. ${ }^{32}$ Este tipo de câncer representa um grave problema de saúde pública nos países da América Latina, considerada como uma das regiōes de maior incidência no mundo. ${ }^{33-35}$ Cabe destacar o percentual significativo de mulheres que nunca realizaram o exame colpocitológico nos países da América Latina, provavelmente aquelas de maior risco. ${ }^{34} \mathrm{Em}$ nosso país, Distrito Federal, Goiânia e Belém apresentaram as maiores taxas de incidência anuais ajustadas por idade deste câncer, com valores de 50,7/100.000, 41,1/100.000 e 34,7/100.000, respectivamente. ${ }^{9}$ Estudo recente conduzido na cidade de São Paulo demonstrou uma discreta redução na taxa de mortalidade por câncer de colo de útero, o que pode estar relacionado ao aumento na cobertura do rastreamento desta doença através do método de papanicolaou. ${ }^{36}$ No Paraná, uma clara diminuição da mortalidade por câncer cervical foi observada a partir da introdução de um programa de rastreamento, com aumento na cobertura do exame colpocitológico de $43 \%$ para cerca de $86 \%$ da população feminina adulta local, em um período de apenas cinco anos de funcionamento deste programa. ${ }^{37} \mathrm{~A}$ infecção por HPV associada a outros fatores de risco, como história de outras doenças sexualmente transmissíveis, tabagismo e uso de contraceptivo oral, representa importante papel na progressão das lesões escamosas intraepiteliais para a malignidade em mulheres brasileiras. ${ }^{38}$

\section{Câncer de mama}

$\mathrm{Na}$ atualidade, o câncer de mama é considerado como o segundo tipo de câncer mais comum no mundo, sendo o mais freqüente entre as mulheres. Ao contrário do câncer de colo de útero, esta doença encontra-se relacionada ao processo de industrialização, com risco de adoecimento associado a elevado status sócioeconômico, além de outros fatores de risco clássicos descritos, tais como baixa paridade, idade precoce da menarca e tardia da menopausa, obesidade, altura e consumo de álcool. ${ }^{6}$ Recentemente, este tipo de câncer 
vem se transformando em um importante problema de saúde pública na América Latina, tendo sido observado, nesta região, um aumento consistente nas taxas de mortalidade por câncer de mama nos últimos quarenta anos. ${ }^{39}$ Estudo conduzido no estado de São Paulo identificou o câncer de mama como a principal causa de mortalidade por neoplasias em mulheres na faixa etária de 30 a 49 anos, no período de 1991 a $1995 . .^{40}$ De acordo com estudo caso-controle conduzido em hospital universitário de Belo Horizonte, o perfil de risco para o câncer de mama relativo a fatores sócioeconômicos e reprodutivos apresentou-se similar em mulheres com diagnóstico efetuado na pré e na pósmenopausa. ${ }^{41}$ A partir de estudo ecológico realizado com dados de exposição a pesticidas em onze estados do Brasil, foram identificadas correlaçōes importantes entre esta exposição e distúrbios no sistema reprodutivo humano, incluindo a mortalidade por câncer de mama, principalmente em mulheres com idade entre 50 a 69 anos no período de 1995 a 1997.42 No entanto, estudo caso-controle realizado por Mendonça et al. (1999) ${ }^{43}$ na Região Metropolitana do Rio de Janeiro, incluindo 177 casos e 350 controles, não verificou associação entre nível sérico de organoclorados e aumento de risco para o câncer de mama. Com relação ao rastreamento das neoplasias malignas de mama, observou-se que mulheres idosas e mulheres com baixo grau de escolaridade apresentaram menores oportunidades de diagnóstico precoce no município de Botucatu, São Paulo. ${ }^{44}$ As mais elevadas taxas de incidência anuais de câncer de mama no Brasil, ajustadas por idade e por 100 mil mulheres, foram encontradas em São Paulo $(94,0)$, Distrito Federal $(86,1)$ e Porto Alegre $(66,5) .{ }^{9}$

\section{Câncer de próstata}

O câncer de próstata também se sobressai como um importante problema de saúde pública mundial, tendo sido observado, a partir de 1960, um aumento progressivo na sua incidência em vários países. ${ }^{45}$ Embora exaustivamente pesquisado, o papel dos fatores ambientais na gênese do câncer de próstata permanece, ainda, não muito bem compreendido, sendo enfatizado por alguns estudos a associação desta doença com componentes dietéticos específicos, como maior risco associado ao consumo de gorduras e carnes. ${ }^{6}$ No Brasil, pesquisas têm sido desenvolvidas na tentativa de se esclarecer a associação entre câncer de próstata, além de outros tipos de câncer, e fatores ambientais e/ou ocupacionais. ${ }^{8,46,47}$ Vale destacar que, em estudo conduzido com a finalidade de verificar as diferenças no perfil de mortalidade proporcional por câncer em militares da Marinha do Brasil, o câncer de próstata apresentou-se como uma das três causas de maior mortalidade na população estudada, sendo mais comum em militares do que na população geral de referência. ${ }^{47}$ Dados recentes do Ministério da Saúde (2003) ${ }^{9}$ revelaram que as maiores taxas anuais de incidência ajustadas por idade por 100 mil homens encontram-se no Distrito Federal $(112,1)$, Goiânia $(99,3)$ e São Paulo $(86,4)$. Apesar de o aumento na utilização do teste do Antígeno Prostático Específico (PSA), principalmente a partir da década de 90 , ser apontado como o principal fator para o maior registro de casos novos de câncer de próstata, existem, ainda, muitas controvérsias no que se refere à introdução deste teste para rastreamento organizado em nível populacional. ${ }^{45}$

\section{Câncer de cólon e reto}

O câncer de cólon e reto relaciona-se a fatores ambientais, especialmente dietéticos, à predisposição genética e à obesidade, entre outros fatores, sendo que, no Brasil, os maiores valores de taxas médias anuais de incidência ajustadas por idade foram encontrados no Distrito Federal (25,5/100.000 em homens e 22,8/ 100.000 em mulheres) e Porto Alegre (22,3/100.000 em homens e 17,7/100.000 em mulheres). ${ }^{9}$ No período de 1980 a 1993, foi constatado um aumento significativo da taxa de mortalidade padronizada por câncer de cólon e reto em áreas industrializadas da Baixada Santista, região Sudeste do país, em relação a áreas não industrializadas, sinalizando para a associação entre este tipo de câncer e fatores ambientais, como hábitos de vida, condições ocupacionais e características urbanoindustriais. ${ }^{48}$ Embora seja um achado contraditório em relação à carcinogênese do câncer de cólon, observouse uma associação negativa entre este câncer e a presença de megacólon, patologia muito comum em algumas regiōes do Brasil, freqüentemente associada à Doença de Chagas, e que cursa com constipação crônica importante. ${ }^{49}$ Considerando os preceitos teóricos relativos a relevância epidemiológica, possibilidade de prevenção e diagnóstico precoce com apropriada relação custo-benefício e capacidade de alteração do curso natural da doença com o tratamento, Lotufo (2003) enfatizou que as práticas de saúde pública em nosso país devem contemplar açôes mais direcionadas ao câncer de cólon e reto. ${ }^{50}$ 


\section{CONCLUSÕES}

A avaliação do risco de câncer no Brasil deve sempre levar em consideração a dificuldade dos registros de câncer existentes no país para manter a coleta de dados continuamente. $\mathrm{Na}$ maioria das vezes, estes registros enfrentam problemas estruturais para a sua manutenção, o que pode levar a vieses que se refletirão na análise dos dados das diferentes regióes do país. Todavia, o quadro de risco atual do câncer no Brasil e suas tendências mostram relevância no âmbito da saúde pública e evidenciam a necessidade contínua de realização de pesquisas sobre este tema, as quais são essenciais para o desenvolvimento de políticas de saúde adequadas que visem ao controle de câncer no país.

Alguns aspectos relativos ao comportamento dos principais tipos de câncer no panorama brasileiro merecem ser enfatizados, a saber: progressivo aumento da incidência do câncer de pulmão, especialmente em mulheres, em função do aumento da exposição deste grupo etário ao tabaco, o que influencia também a incidência dos cânceres de boca e faringe, laringe e esôfago; associação observada entre hábitos alimentares comuns em determinadas regiōes do país e risco de câncer específico, como consumo de mate muito quente e de churrasco e risco de câncer de esôfago e consumo diário de carne e baixa ingestão de frutas e risco de câncer de estômago; papel da infecção por HPV e da história de outras doenças sexualmente transmissíveis no risco de câncer de colo de útero, além da importância do aumento da cobertura do exame colpocitológico na diminuição da mortalidade por este tipo de câncer no Brasil; importância do câncer de mama como principal causa de morte por neoplasias em mulheres no país e questōes sócio-econômicas, reprodutivas e ambientais que podem estar relacionadas ao risco e sobrevida locais; relação de fatores ambientais com o risco de câncer de próstata e câncer de cólon e reto no país.

Em função do contexto apresentado, destacamos a importância das atividades de prevenção primária, tais como controle do uso de tabaco, redução do consumo de álcool e estímulo à dieta com qualidade nutricional e à prática de atividades físicas regulares, com a finalidade de eliminação ou diminuição, de maneira eficiente, eficaz e efetiva, dos fatores de risco associados ao câncer e a várias outras doenças crônicas não-transmissíveis. Além disto, as estratégias de prevenção secundária para alguns tipos de câncer que, na experiência de outros países, tiveram impacto na mortalidade, como, por exemplo, para os cânceres de mama e de colo uterino, devem se tornar políticas prioritárias. Uma efetiva política de controle do câncer em nosso país deve considerar também a situação sócio-econômica e as desigualdades existentes nas diversas regiōes do Brasil, sendo que todas as ações programáticas devem se dar no contexto da prevenção de doenças crônicas nãotransmissíveis e de promoção da saúde.

\section{REFERÊNCIAS}

1. World Health Organization. Policies and managerial guidelines for national cancer control programs. Rev Panam Salud Publica. 2002 Nov;12(5):366-70.

2. Waters WF. Globalization, socioeconomic restructuring, and community health. J Community Health. 2001 Apr;26(2):79-92.

3. Laurenti R. Transição demográfica e transição epidemiológica. Anais do $1^{\circ}$ Congresso Brasileiro de Epidemiologia; 1990 set 2-6; Campinas, Brasil. Rio de Janeiro: Abrasco; 1990. p. 143-65.

4. Albala C, Vio F, Yanez M. Transición epidemiológica en América Latina: comparación de cuatro países. Rev Med Chil. 1997 Jun;125(6):719-27.

5. Susser M, Susser E. Um futuro para a epidemiologia. In: Almeida Filho N, Barreto ML, Veras RP, Barata RB. Teoria epidemiológica hoje: fundamentos, interfaces, tendências. Rio de Janeiro: Abrasco; 1998. p.187-212. (Série Epidemiológica 2).

6. Parkin DM, Bray FI, Devesa SS. Cancer burden in the year 2000. The global picture. Eur J Cancer. 2001 Oct;37 Suppl 8:S4-66.

7. Albala C, Vio F, Kain J, Uauy R. Nutrition transition in Latin America: the case of Chile. Nutr Rev. 2001 Jun;59(6):170-6.

8. Koifman S, Koifman R. Environment and cancer in Brazil: an overview from a public health perspective. Mutat Res. 2003 Nov;544(2-3):305-11.

9. Instituto Nacional de Câncer; Ministério da Saúde. Câncer no Brasil: dados dos registros de base populacional, vol 3 . Rio de Janeiro (Brasil): INCA; 2003.

10. Pisani P, Bray F, Parkin DM. Estimates of the world-wide prevalence of cancer for 25 sites in the adult population. Int J Cancer. 2002 Jan 1;97(1):72-81.

11. Ministério da Saúde. Informaçōes de saúde: estatísticas vitais/ mortalidade geral [homepage na Internet]. [citado em 28 jul 2004]. Disponível em: http://www.datasus.gov.br/.

12. Wunsch Filho V, Moncau JE. Mortalidade por câncer no Brasil 1980-1995: padrões regionais e tendências temporais. Rev Assoc Med Bras. 2002 jul-set;48(3):250-7.

13. Parkin DM, Bray F, Ferlay J, Pisani P. Estimating the world cancer burden: Globocan 2000. Int J Cancer. 2001 Oct 15;94(2):153-6.

14. da Costa e Silva VL, Koifman S. Smoking in Latin America: 
a major public health problem. Cad Saúde Pública. 1998;14 Suppl 3:S99-108.

15. Algranti E, Menezes AM, Achutti AC. Lung cancer in Brazil. Semin Oncol. 2001 Apr;28(2):143-52.

16. Menezes AM, Horta BL, Oliveira AL, Kaufmann RA, Duquia R, Diniz A, et al. Risco de câncer de pulmão, laringe e esôfago atribuível ao fumo. Rev Saúde Pública. 2002 Apr;36(2):129-34.

17. Wunsch-Filho V. The epidemiology of oral and pharynx cancer in Brazil. Oral Oncol. 2002 Dec;38(8):737-46.

18. Franceschi S, Bidoli E, Herrero R, Munoz N. Comparison of cancers of the oral cavity and pharynx worldwide: etiological clues. Oral Oncol. 2000 Jan;36(1):106-15.

19. Antunes JL, Biazevic MG, de Araujo ME, Tomita NE, Chinellato LE, Narvai PC. Trends and spatial distribution of oral cancer mortality in Sao Paulo, Brazil, 1980-1998. Oral Oncol. 2001 Jun;37(4):345-50.

20. Goldenberg D. Mate: a risk factor for oral and oropharyngeal cancer. Oral Oncol. 2002 Oct;38(7):646-9.

21. Schlecht NF, Pintos J, Kowalski LP, Franco EL. Effect of type of alcoholic beverage on the risks of upper aerodigestive tract cancers in Brazil. Cancer Causes Control. 2001 Sep;12(7):579-87.

22. Castellsagué X, Muñoz N, de Stefani E, Victora CG, Quintana MJ, Castelletto R, et al. Smoking and drinking cessation and risk of esophageal cancer (Spain). Cancer Causes Control. 2000;11(9):813-8.

23. Castellsagué X, Muñoz N, De Stefani E, Victora CG, Castelletto R, Rolón PA, et al. Independent and joint effects of tobacco smoking and alcohol drinking on the risk of esophageal cancer in men and women. Int J Cancer. 1999 Aug 27;82(5):657-64.

24. Parkin DM, Whelan SL, Ferlay J, Teppo L, Thomas DB. Cancer incidence in five continents, vol 8. Lyon: IARC; 2002. (IARC Scientific Publications; no. 155).

25. Lourenco LG, Hamada GS. Gastric cancer in Brazil. Gastric Cancer. 2001;4(2):103-5.

26. Hamada GS, Kowalski LP, Nishimoto IN, Rodrigues JJ, Iriya K, Sasazuki S, et al. Risk factors for stomach cancer in Brazil (II): a case-control study among Japanese Brazilians in Sao Paulo. Jpn J Clin Oncol. 2002 Aug;32(8):284-90.

27. Nishimoto IN, Hamada GS, Kowalski LP, Rodrigues JG, Iriya K, Sasazuki S, et al. Risk factors for stomach cancer in Brazil (I): a case-control study among non-Japanese Brazilians in Sao Paulo. Jpn J Clin Oncol. 2002 Aug;32(8):277-83.

28. Verdecchia A, Mariotto A, Gatta G, Bustamante-Teixeira MT, Ajiki W. Comparison of stomach cancer incidence and survival in four continents. Eur J Cancer. 2003 Jul;39(11):1603-9.

29. Pillai MR, Lakshmi S, Sreekala S, Devi TG, Jayaprakash PG, Rajalakshmi TN, et al. High-risk human papillomavirus infection and E6 protein expression in lesions of the uterine cervix. Pathobiology. 1998;66(5):240-6.

30. Munoz N. Human papillomavirus and cancer: the epidemiological evidence. J Clin Virol. 2000 Oct;19(1-2):1-5.

31. Franco EL, Duarte-Franco E, Ferenczy A. Cervical cancer: epidemiology, prevention and the role of human papillomavirus infection. CMAJ. 2001 Apr 3;164(7):1017-25.

32. Eluf-Neto J. Number of sexual partners and smoking behaviour as risk factors for cervical dysplasia: comments on the evaluation of interaction. Int J Epidemiol. 1994 Oct;23(5):1101-4.

33. Novoa Vargas A, Echegollen Guzman A. Epidemiología del cáncer de cérvix en Latinoamérica. Ginecol Obstet Mex. 2001;69(6):243-6.

34. Eluf-Neto J, Nascimento CM. Cervical cancer in Latin America. Semin Oncol. 2001 Apr;28(2):188-97.

35. Arrossi S, Sankaranarayanan R, Parkin DM. Incidence and mortality of cervical cancer in Latin America. Salud Publica Mex. 2003;45 Suppl 3:S306-14.

36. Fonseca LA, Ramacciotti Ade S, Eluf Neto J. Tendência da mortalidade por câncer do útero no Município de São Paulo entre 1980 e 1999. Cad Saúde Pública. 2004 janfev;20(1):136-42. Epub 2004 mar 8.

37. Bleggi Torres LF, Werner B, Totsugui J, Collaco LM, Araujo SR, Huculak M, et al. Cervical cancer screening program of Parana: cost-effective model in a developing country. Diagn Cytopathol. 2003 Jul;29(1):49-54.

38. Cavalcanti SM, Zardo LG, Passos MR, Oliveira LH. Epidemiological aspects of human papillomavirus infection and cervical cancer in Brazil. J Infect. 2000 Jan;40(1):80-7.

39. Robles SC, Galanis E. Breast cancer in Latin America and Caribbean. Rev Panam Salud Publica. 2002 Mar;11(3):178-85.

40. Haddad N, da Silva MB. Mortalidade por neoplasmas em mulheres em idade reprodutiva - 15 a 49 anos - no estado de São Paulo, Brasil, de 1991 a 1995. Rev Assoc Med Bras. 2001;47(3):221-30.

41. Gomes AL, Guimaraes MD, Gomes CC, Chaves IG, Gobbi $\mathrm{H}$, Camargos AF. Risk factors for breast cancer among preor post-menopausal women in Belo Horizonte, Brazil. Gynecol Obstet Invest. 2001;52(3):173-9.

42. Koifman S, Koifman RJ, Meyer A. Human reproductive system disturbances and pesticide exposure in Brazil. Cad Saúde Pública. 2002;18(2):435-45. Epub 2002 ago 16.

43. Mendonça GA, Eluf-Neto J, Andrada-Serpa MJ, Carmo $\mathrm{PA}$, Barreto $\mathrm{HH}$, Inomata ON, et al. Organochlorines and breast cancer: a case-control study in Brazil. Int J Cancer. 1999 Nov 26;83(5):596-600. 
44. Molina L, Dalben I, de Luca LA. Análise das oportunidades de diagnóstico precoce para as neoplasias malignas de mama. Rev Assoc Med Bras. 2003;49(2):185-90. Epub 2003 jul 22.

45. Quinn M, Babb, P. Patterns and trends in prostate cancer incidence, survival, prevalence and mortality. Part II: individual countries. BJU Int. 2002 Jul;90(2):174-84.

46. Meyer A, Chrisman J, Moreira JC, Koifman S. Cancer mortality among agricultural workers from Serrana Region, state of Rio de Janeiro, Brazil. Environ Res. 2003 Nov;93(3):264-71.

47. Silva M, Santana VS, Loomis D. Mortalidade por câncer em militares da Marinha do Brasil. Rev Saúde Pública. 2000;34(4):373-9.
48. Medrado-Faria MA, Rodrigues de Almeida JW, Zanetta DM. Gastric and colorectal cancer mortality in an urban and industrialized area of Brazil. Rev Hosp Clin Fac Med São Paulo. 2001;56(2):47-52.

49. Garcia SB, Aranha AL, Garcia FR, Basile FV, Pinto AP, de Oliveira EC, Zucoloto S. A retrospective study of histopathological findings in 894 cases of megacolon: what is the relationship between megacolon and colonic cancer? Rev Inst Med Trop São Paulo. 2003;45(2):91-3. Epub 2003 maio 14.

50. Lotufo PA. The noise stops you from hearing good music: the possibilities for a mortality reduction program for cancer of the colon and rectum in Sao Paulo. São Paulo Med J. 2003 maio 5;121(3):95-6. Epub 2003 ago 8. 